\title{
Collaborative Refinement of Emergency Plans through Public Engagement
}

\author{
$\mathrm{M}^{\mathrm{a}}$ Carmen Penadés ${ }^{1}$, Marcos Borges ${ }^{2}$, Adriana Vivacqua ${ }^{2}$, José H. Canós ${ }^{1}$, Carlos Solis ${ }^{3}$ \\ ${ }^{1}$ ISSI-DSIC \\ Universitat Politècnica de València \\ 46022-Valencia (Spain) \\ \{mpenades, jhcanos\}@dsic.upv.es \\ ${ }^{2}$ Graduate Program in Informatics- \\ Instituto de Matematica \\ Federal University of Rio de Janeiro \\ Rio de Janeiro, Brasil \\ mborges@nce.ufrj.br; \\ avivacqua@dcc.ufrj.br \\ ${ }^{3}$ Lero, the Irish Software \\ Engineering Research Centre \\ University of Limerick \\ Limerick, Ireland \\ Carlos.Solis@lero.ie
}

\begin{abstract}
Emergency plans are fundamental for the speedy and effective response in disaster situations. Plans are often constructed by teams of experts, who apply their expertise to define response procedures, but lack part of location-specific knowledge that can be very relevant to make decisions during responses. Such knowledge is, however, in the minds of people who use those spaces every day, but are not involved in the planning processes. In this paper, we advocate for citizens' involvement in emergency plan elaboration via Public Participation, a mechanism long time used in other areas of egovernment. We define the steps of a collaborative process for the elicitation of citizen's knowledge via Public Participation. A summary of the results of an initial case study is used to demonstrate the feasibility of our proposal for improving emergency plans.
\end{abstract}

Keywords - public consultation, collective knowledge, emergency management

\section{INTRODUCTION}

Emergency response is one of the most critical activities performed by humans: it involves processes where decisions are based on information coming from different sources, which must be accessed and combined adequately to avoid both information gaps and overload. The basis of all decision making and action during emergency response is the emergency response plan (or emergency plan, for short), a document that includes procedures to be executed in response to an incident, and some the information required to make decisions (such as maps, pictures, videos, etc). The plan directs responders towards the event location, defines the procedures to apply in response to each possible incident, and provides the information needed to perform the response actions.

However, having a plan may not be enough. As pointed out by Palen et al. [1], a critical part of the response efforts lies with the individuals affected by the emergency. As a matter of fact, in most cases the first response actions are performed by in-place victims themselves. They are close to the site and their actions may make rescue efforts easier or harder depending on what actions they did. Unfortunately, citizens are usually not well acquainted with emergency plans, missing knowledge that may be helpful in case of crises. While respondents get from the plan technical knowledge of an emergency site and response procedures, the individuals living in the area have a different type of knowledge: as they inhabit these spaces on a daily basis, they will likely develop an instinctive response to emergency situations based on their daily interactions with the environment (they have knowledge different from, and possibly more accurate than, the knowledge contained in the plan). Thus, we believe that potentially valuable information for emergency response is available from the individuals involved in the emergency, and should therefore be gathered from them.

Our goal in this work is twofold: on one hand, to increase citizen's acquaintance with emergency plans; on the other hand, to investigate and devise alternative ways to obtain additional information during the planning process, via the participation of citizens in the early evaluation of the plans. To achieve this goal, we rely on principles of Public Participation, a mechanism to acquire knowledge from individuals using different strategies such as panels, surveys, public hearings, and others [2]. Specifically, we claim that the implementation of a public consultation process that exposes the content of the emergency plan to citizens may lead to an overall improvement of the plan and a higher familiarity of individuals with it. The citizens can generate important contextual information that, having a non-expert origin, may lead an improvement of the plans and safer conditions long before the occurrence of actual emergencies.

In this paper, we take first steps towards a process for Public Participation for plan improvement. We devised a collaborative knowledge elicitation process that would also help participants reflect of the problem and learn something of the domain. We ran a case study using three different tools for gathering public feedback on emergency plans. Results show that regular individuals (non-expert) had relevant information pertaining to emergency plan improvement. They are not only able of providing useful information, they also became aware of what they did not know through interaction and reflection, and suggested improvements for plan dissemination and security design, improving preparedness overall.

This paper is structured as follows. In the next section, we present background knowledge. In section 3, we describe our collaborative proposal for improving emergency plans based

The work of M.C. Penadés and J.H. Canós is partially funded by the Spanish MEC, under grant TIPEx (TIN2010-19859-C03-03). The work of M.Borges is partially funded by grants No 560223/2010-2 and 304252/2008-5 from CNPq (Brazil). The work of A.S. Vivacqua is partially funded by FAPERJ (Brazil). The work of C. Solís is funded by Science Foundation

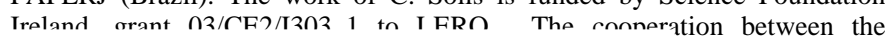


on Public Participation. The case study is described in section 4, which includes information about the studies conducted and results obtained. Finally, conclusions and future work.

\section{A PRIMER ON PUBLIC PARTICIPACION PROCESSES}

Public Participation has received much attention in the last 20 years, with numerous publications related with different aspects such as types of Public Participation processes [2][3], frameworks for their evaluation [4], and experiences in their definition and application in different fields such as environmental policy [5], or urban planning [6]. Several common characteristics of Public Participation processes have been identified by Creighton [5]. First, they are not intended to be mere information sources; rather, they are interactive, creating a bidirectional flow of information to and from individuals. Second, they are not accidental: they are designed, planned and executed by organizations interested in knowing the public opinion. And third, some relevant role is reserved to the feedback provided by public in the final decision being made by the organization. There are numerous types of Public Participation processes, which can be classified following different criteria [1][7]. Specifically, deliberative methods are those where a selected group of individuals are gathered to participate in meetings where discussions about a specific issue are held, that result in, a decision. Examples of deliberative processes are citizen juries, panels, planning cells and deliberative polling. They differ from each other on the size of groups, the meetings schedule, and the type of outcome expected. Conversely, non-deliberative methods are those where individuals are not requested to reach a group conclusion, but to provide some feedback individually. Examples are surveys, public hearings, open houses, and citizen advisory committees, among others. The differences arise in the size of the group of study, the goal of the process and the way the interaction with the individuals is arranged.

Collaborative tools may be used to support deliberative processes without need to hold physical meetings [8]. As a consequence, new models and implementations of Public Participation processes are appearing; for instance, Webenabled collaborative tools can support new implementations of Public Participation processes.

\section{PUBLIC PARTICIPATION FOR EMERGENCY PLAN IMPROVEMENT}

Rowe and Frewer [4] discussed the relevance of public feedback in risk identification and management for science and technology policy. They pointed out that citizens' views of risks often were different that the one of risk management experts, and considered that public feedback offers valuable information in several stages of the risk management process. We have extrapolated this conclusion to the emergency management field, with the aim of taking advantage of citizens' contribution at the different stages of the emergency management lifecycle. So far, most of the attempts to use public generated knowledge have focused on the response phase, with numerous studies about the use of social networks as the main communication channel (see e.g., http://idisaster. wordpress.com/bibliography/). However, Public Participation in other stages of the emergency lifecycle, especially in planning, has been little explored.
We believe that non-expert, local knowledge can bring new insight to emergency planning processes. As a consequence, we wanted to study how citizens potentially involved in an emergency can cooperate in the improvement of emergency plans by providing feedback right after their elaboration. Doing so, we hope that significant pieces of previous personal knowledge become part of the emergency plan. Besides emergency planners, who design and implement emergency plans, citizens that inhabit in the plan's area of influence get involved in planning via the Public Participation processes. Like responders, citizens have personal knowledge that they use in the Public Participation activities, but this knowledge has a different nature. To support our discussion, we distinguish two types of previous personal knowledge, namely expert and naïve knowledge. The former is mostly present in responders mind due to their background and training on emergency responses, whereas the latter is mostly related with "local" aspects related to the specific area the citizens live in. Of course, both knowledge sets may overlap in some cases.

The emergency plan is initially built by planners, and is exposed to citizens who can, in turn, produce feedback that may eventually be used by planners to improve the plan.

\section{A. A process for eliciting information from the public}

Fig. 1 shows the process we defined to elicit naïve knowledge. A frequent issue with emergency response is that no one really worries about an emergency until it actually happens. This makes it harder for people to respond when it does happen. Thus, the process starts with a Familiarization subprocess, in which citizens are invited to think about how they would act in response to an emergency. With this aim, emergency planners design a set of emergency scenarios and a set of related questions to capture the citizens' response. The familiarization is closely followed by a Reflection subprocess, where citizens think about their selected actions; specifically, they are requested to give alternatives different to the ones provided in the emergency plan. In the Critique subprocess, citizens provide direct feedback on the emergency plans. They use what they have learned and reflected about, as well as their newly acquired awareness of these situations, to give critical comments on the plan itself (add new and/or reply to critical comments about the plan). Next, the participants make a final

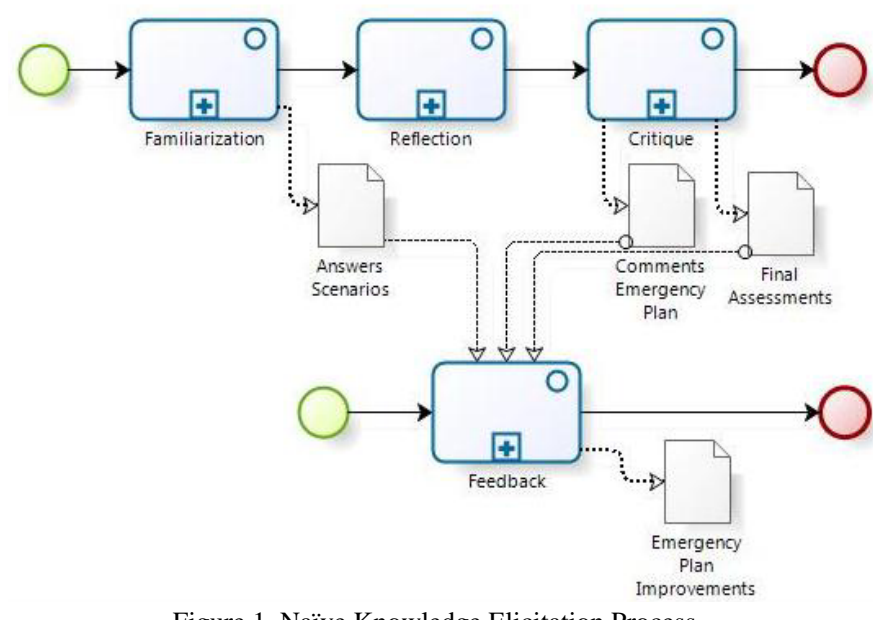

Figure 1. Naïve Knowledge Elicitation Process 
assessment about all the activities performed in the process. Given the amount of information that may be generated and the potentially large number of people involved, it is highly advisable for this process to be supported by group interaction tools. Finally, in Feedback subprocess, all comments are translated into feedback (usually through some processing, aggregation or statistics) so they can be given to planners for improvement of the plans.

\section{A CASE STUDY}

To verify the suitability of the knowledge elicitation process proposed, we designed an experimental study based on it. Our goal was not only to enrich the emergency plan, but also to test the process defined and its effect on participants, checking in this way the ability of individuals to provide feedback about emergency plans. We engaged a total of 45 Computer Science students of the Universitat Politècnica de València. They were first requested to provide non-expert comments on the emergency plan of the main building of the School of Computer Science, where they usually attend to classes. While they had extensive experience with computers, they weren't knowledgeable in emergency management. Given the length of the emergency plan, we decided to constrain the study to some parts of the plan, involving only a few emergency situations.

\section{A. Study Design and Implementation}

To familiarize participants with emergency situations, we designed an exercise in which participants were given emergency scenarios to respond to. Specifically, we provided seven scenarios: "a fire starts in classroom", "you discover a fire at the ground floor Study Room”, "a classmate faints”, "the building's alarm rings", "the building's alarm rings while you are in the lift", "strong explosion in the building", and "collective panic". The participants were asked about what they would/wouldn't do in each situation, which exit way they would take and whether they would use any safety equipment (such as fire extinguishers) or not. Scenarios were designed to be realistic, that is, they included events that could possibly happen. They were described in a simple way, so that participants wouldn't have trouble understanding them. Forms designed with Google Docs were used to implement the process of collecting information on each participant's individual actions.

In the Reflection subprocess, participants were given parts of the current emergency plan that concerned the scenarios they had responded to. After revising the reduced emergency plan, each participant was asked to comment on it. The questions were about what information would they add to or remove from the plan, what inconsistencies had they detected between the information in the plan and the actual building state (e.g. were the maps up to date? Had the building the appropriate signage? Existence of extinguishers, alarms, etc.); and finally, to add any comment about the plan that they considered important. Participants were divided into three groups of 15, and each group used a different collaborative tool to consult the information about the emergency plan and provide feedback:
Blog: Information was pasted into a blog, built with Blogger (http://experimentoparteb.blogspot.com/) where participants could provide comments in response to what they read. Comments were identified and visible to others, who were allowed to respond.

Annotations: The tool used was A.nnotate, an online annotation, collaboration and indexing system for documents. Information was published at http://a.nnotate.com/php/pdf notate.php?d=2011-03-01\&c=Nu4NgnDY, as a set of images and the participants could post notes containing their comments (similar to stickies). Notes were identified and visible by all participants, who could comment on each other's notes.

Spatial Hypertext: We used ShyWiki [9], a wiki which uses spatial hypertext. ShyWiki manages a network of wiki pages whose content is spatially organized: notes may be placed in different regions of the page, moved around, and may be different sizes and colors. The notes can contain text, hyperlinks, and images. Information was pasted onto the wiki at http://gaia.lero.ie:8080/ShyWiki10/ and participants could post annotations onto them. Again, comments were identified and visible by all, so responses could be added.

Finally, the participants concluded the Critique subprocess answering a global evaluation form with questions with multiple choice responses. We asked them whether their initial answers (their instinctive reactions to scenarios proposed) were agreed with the specified in the emergency plan or not; we also asked about the perceived usefulness of the emergency plans, if their participation in the experiment has increased their emergency preparedness, and finally, if they thought that their comments could help to improve the current emergency plans. Participants were also allowed to add comments after responding to the questionnaire. Such comments were very interesting, being actually a source of important information to be discussed with emergency managers at the campus.

\section{B. A summary of the results}

We present an analysis of responses received using the three different collaborative tools in the critique subprocess: (a) in the blog, a total of 57 comments were made about the emergency plan provided; (b) in A.nnotate, 88 comments were made on it; and, (c) on ShyWiki, a total of 55 comments was made, many of which chained together (comments to previous comments), as we perceived in A.nnotate.

Fig. 2 shows a screenshot of A.nnotate tool that concerns the fire and evacuation response procedure. In this case, there are 51 comments grouped into 14 notes. The participants can reply to any note adding new comments about some item. For instance, the expanded note framed in dashed lines in the right site is about the item "phone numbers of whom to call (clerks's desk, in this case) when a fire starts or is discovered". There are nine replies to discuss it. The general comment is that phone numbers of the clerks' desk are not clearly published in any public location. Some suggestions were that the phone numbers, as well as the emergency alarm activation button should be in the panels placed near the fire extinguishers.

As a whole, the nature of comments did not vary widely between the different tools, but the type of interaction did. In blog style comments, there were fewer interactions and 


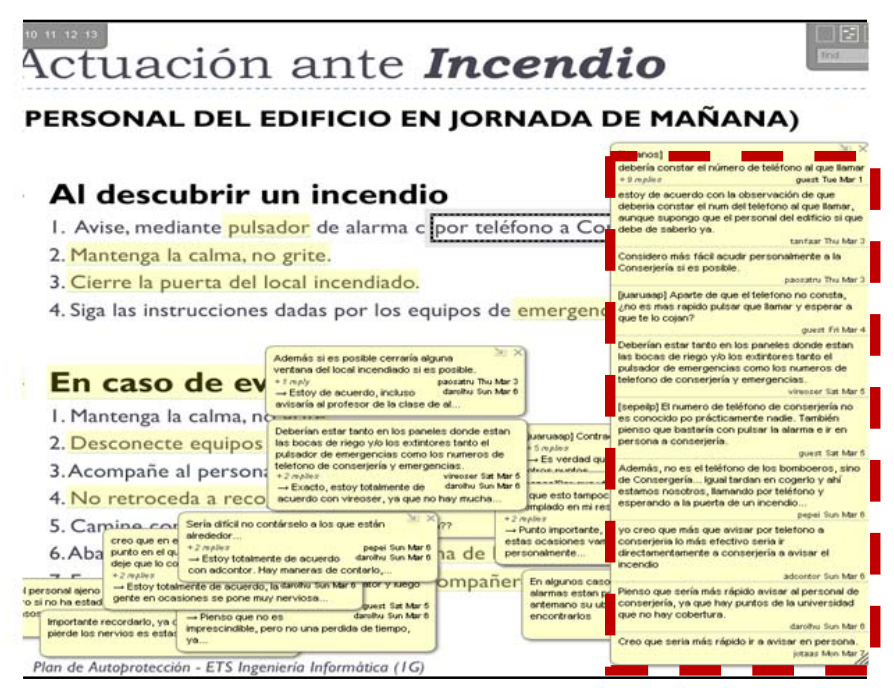

Figure 2. Screenshot of A.nnotate showing participants' comments about fire and evacuation response procedure.

comments on other comments, while in annotation based tools, comments were plentiful and better organized in threads. We speculate that this may be due to the fact that participants can more easily see the annotations displayed over the plan and respond to them, creating annotation threads. A.nnotate users made significantly more comments; we assume that it was because the tool is better finalized and easier to use.

An important aspect is how these comments are translated into feedback to planners for improvements of the plans. Annotation based tools (A.nnotate and ShyWiki) are better than the blog, because all the related comments are in the same annotation threads and it is easier to process. Further analysis and experiments are planned in this respect.

\section{Global Assessment}

Through participation in the activity, participants learned about emergency plans, and realized how much knowledge they had about emergency situation. This shows that our activities in familiarization subprocess reached their goal. Students became more aware of the problems and issues of emergencies, and were able to make useful comments and suggestions that would lead to improve the emergency plans. This goes to show that Public Participation is not a one-way street, where participants provide information or complain about a given issue, but can also serve as a two way learning experience for participants, as it induces reflection and thought.

At the end of the Critique subprocess, we analyzed scenarios and comments provided relating to each section of the emergency plan. Looking at the number of comments and their distribution through the emergency plan, we could observe that there was greater involvement in the response procedures relating to more concrete incidents, such as fire or medical emergencies. The participants suggested improvements in many directions. However, for building collapse and explosions, response procedures are more complex and there may be many causes for an incident, so participants provided less feedback and suggestions for improvements.

\section{CONCLUSIONS}

In this paper, we explored the use of Public Participation techniques to improve emergency plans by using citizens' knowledge as a complement to the one of experts. Citizens' knowledge is relevant because they have contextual knowledge of the sites. Through a study with 45 participants, we noticed that they provided useful comments for improving a concrete emergency plan. Some of them noticed situations that were unknown to the planners. The adoption of three different collaborative tools allowed us to see how effective they were for this end. While this study was not specifically designed to evaluate or compare them, we could already notice that there were certain differences in responses and the way in which participants interacted. This is an important observation, as it indicated that certain tool characteristics will better fit Public Participation. Based on these observations, we will design new studies and experiments to assess different tools, their strengths and weaknesses.

The next step in our research will be to meet with emergency planners and respondents and show them comments and responses of the study, to elicit what types of changes could be made to the emergency plan.

\section{ACKNOWLEDGMENT}

The authors would like to thank the students of the School of Computer Science at the Technical University of Valencia who participated in this study.

\section{REFERENCES}

[1] Palen, L., Hiltz, S. R. and Liu, S. B. Online forums supporting grassroots participation in emergency preparedness and response. Commun. ACM 50, 3, pp 54-58. March. 2007. DOI $=10.1145 / 1226736.1226766$ http://doi.acm.org/10.1145/1226736.1226766

[2] Abelson J, Forest P-G, Eyles J, Smith P, Martin E and Gauvin F-P. Deliberations about Deliberation: Issues in the Design and Evaluation of Public Consultation Processes, McMaster University Centre for Health Economics and Policy Analysis, Working Paper 01-04, June, 2001

[3] International Association for Public Participation. IAP2 Spectrum of Public Participation. 2007. http://www.iap2.org/associations/4748 /files/spectrum.pdf

[4] Rowe, G. and Frewer, L. J. Public Participation Methods: A Framework for Evaluation. Science, Technology, \& Human Values, Vol. 25 No. 1, pp. 3-29. Sage Publications Inc., 2000

[5] Creighton, J.L. The Public Participation Handbook: Making Better Decisions Through Citizen Involvement. San Francisco, CA: JosseyBass, 2005

[6] Brabham, D.C. Crowdsourcing the Public Participation Process for Planning Projects. Planning Theory, 8; 242, 2009. DOI: 10.1177/1473095209104824

[7] Internet Resources on Public Participation: http://www.epa.gov/international/toolkit/Resources/Links/

[8] Guimarães, Â. Rinaudo, J.-D., Jeffrey, P., Blasques, J., CorralQuintana, S., Courtois, N., Funtowicz, S. and Petit, V. ICT Tools To Support Public Participation In Water Resources Governance \& Planning: Experiences From The Design and Testing of a Multi-Media Platform. Journal of Environmental Assessment Policy and Management. Vol. 5 No. 3. pp. 395-420. 2003

[9] Solis, C. and Ali, N. ShyWiki-A spatial hypertext Wiki. In Proc. of the International Symposium on Wikis. ACM. 2008 